\title{
Improvising New Realities: Movement, Sound and Social Therapeutics
}

\section{Sandra Paola López Ramírez and Chris Reyman}

Focusing on dance and music improvisation, this paper explains the parallels between these practices and the social therapeutic methodology. Social therapeutics is a performative and radically humanistic approach to psychology, therapy, education and community building. ${ }^{1}$ This methodology has helped us further frame our experience of performance and improvisation as powerful tools for social transformation, and we continue to adapt it to empower diverse communities on the U.S.-Mexico border. We structure the paper around the growth and development of the artistic and community-based work of the Institute for Improvisation and Social Action (ImprovISA), ${ }^{2}$ an organization we co-founded in 2011, that is currently based in the sister cities of El Paso, Texas, and Ciudad Juarez, Chihuahua.

On a hot June morning, children pace anxiously in the backstage area waiting to perform in the Víctor Hugo Rascón Banda, the most ornate theater within the biggest performing arts center in Ciudad Juarez, the Centro Cultural Paso del Norte. Earlier that morning, we woke up in our El Paso home, just eighteen miles away from the theater. Even though we are so close, we need to budget at least 1.5 hours for the commute, which involves crossing the U.S.Mexico border on foot, because waiting time on the bridge is unpredictable. This commute has become part of our ritual every time we cross to teach a class or give a workshop, reminding us that the close proximity of these cities stands in stark contrast to the huge gaps in their realities, making evident the differences in access and opportunities between the two countries. This awareness of our privilege has become a contextualizing factor for us; it reminds us of where we come from and why we do this work.

On this day, the state of Chihuahua commissioned the orchestra and contemporary music group of Ccompaz (Citizens Committed with Peace)_an organization that offers afterschool arts-based programs to at-risk youth-to play a concert in commemoration of the International Day Against Drug Abuse and Illicit Trafficking. We have been working with a group of their students for six months and, along with them, we have created two compositions that incorporate improvisation. As the audience members arrive, we gather around our students, who are all dressed up in black suits, white shirts, and black ties, ready for their big debut. For most of them, this is the first time they are performing and for all, the first time they are doing it on the most recognized stage of their city. The youngest of the group, Andrés, a usually rambunctious seven-year-old, is quiet and unsettled. As I (Sandra Paola) approach him to inquire if he is alright, he confesses that he has never done this before and that he doesn't know if he will be able to perform in front of such a large audience. I remind him that his peers, as well as myself and Chris, will be out there performing with him and that it is the group (not the individual members) doing the performance, and therefore he (as an individual) doesn't have to know how to do it.

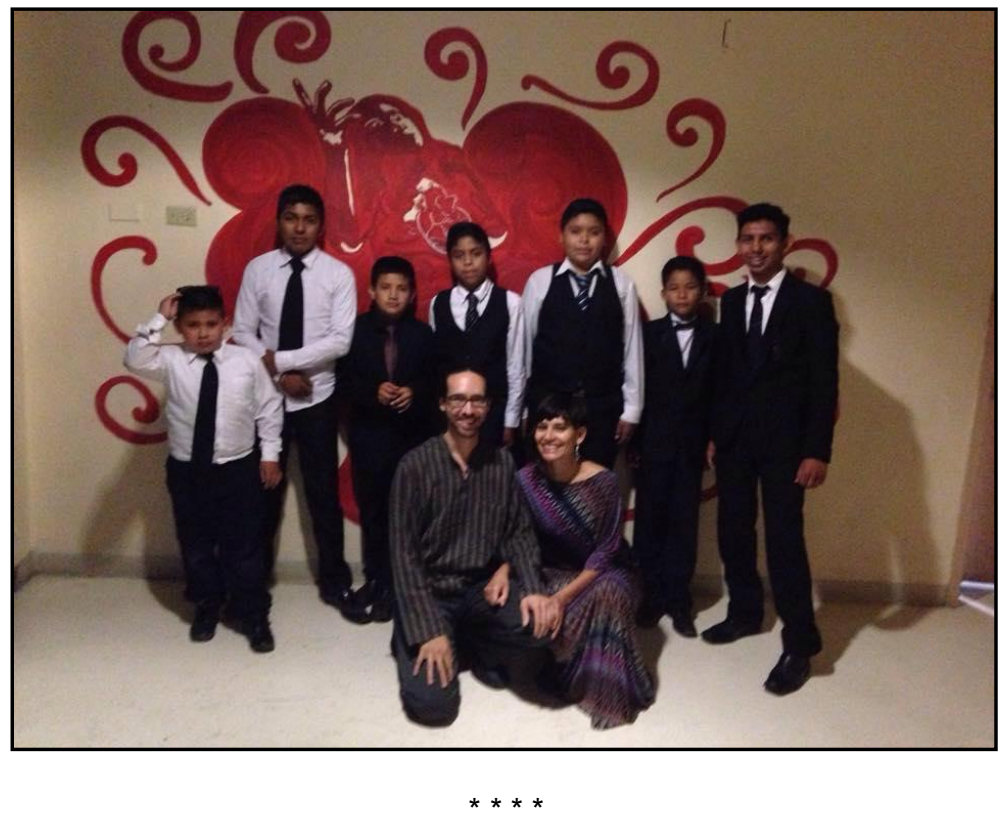

As we walk off the stage after the performance, the applause roaring in the background, I turn to Andrés and ask, "Well, how was it?" To which he responds by jumping victoriously with his hands up in the air while exclaiming, "I 
can't believe I did it!" He is back to his joyous and carefree self again, with (we believe) an added boost of confidence.

\section{Act I: Setting the Stage}

Our work is founded on using improvisation for social change, and it began through our mutual interest in interdisciplinary improvisation. Given who we are and our individual backgrounds, the desire to use it as a tool for social change naturally grew.

\section{Chris}

I developed as a musician primarily through improvisation, and it has remained a constant throughout my life. I spent many hours improvising at the piano, not striving for any particular goal, but being content immersing myself in the creative moment. I was able to be present and let go of my self while improvising, and it wasn't until I developed an interest in Zen meditation that I was able to appreciate these abilities. As I entered college, I maintained an inclination toward progressive approaches to improvisation, and further refined my skills as a graduate student in Jazz Piano Performance at the University of Illinois at Urbana-Champaign. It was the focus on jazz improvisation that led me to struggle with finding sincerity/authenticity in my artistic work, often feeling overwhelmed with expectations of what jazz improvisation was supposed to be and not easily finding my place within this tradition. By the time I finished my doctorate, I felt isolated and uninspired. I decided to take a step away from jazz and started learning to play the accordion, an instrument that my grandfather played, and it was at this time that I began improvising with dancers. I was invited into an interdisciplinary improvisation course at the university, COLAB (Cooperative Laboratory), and also started performing with the professional music-dance collective CompostQ. Immersing myself in interdisciplinary free improvisation was a liberating experience, having no frame of reference as to what it was supposed to be. This opened a whole new world and enabled me to have the freedom that I had experienced playing the piano during my formative years, while helping me to connect with improvisation in a new and refreshing way.

I came to view improvisation as a tool for activism through recognizing the impact it had on my life. For me, improvisation is an escape from the structures of society, a form of protest-not only against conventional modes of music making that favor European classical music, but also against social hierarchies and the stifling education system. Though I didn't have the language to describe it at the time, my years as a university student were often filled with conflict as I advocated for freer forms of improvisation. I have come to see this as a form of activism, and, as an educator, I foster environments for students to improvise and explore, enabling them to create in ways that do not follow traditional notions of what is aesthetically acceptable. Furthermore, the value of improvisation in

\section{Sandra Paola}

I was born and raised in a privileged family in Bogotá, Colombia, a beautiful city marked by stark class divisions and financial inequality. I went to a private bilingual school, had plenty of access to extracurricular activities, and always had the opportunity to travel abroad and experience other cultures. At a very young age, I was introduced to the importance of caring for and sharing with others who weren't as fortunate through a non-profit that my grandfather started. As I grew up, I began to question the reasons for the economic disparities around me and started developing an interest in advocating for the materially poor.

I moved to the U.S. at age nineteen and through many experiences as a 'foreigner,' my eyes were painfully opened. Coming out of my sheltered life, I was suddenly a minority and for the first time, experienced how it felt to be othered. I became politicized and learned to contextualize inequality as part of a complex systemic problem rather than judging an individual's ability to be "moral." Social justice was becoming a bigger focus in my life, and fueled by a new understanding of kindness and compassion through the study of Buddhism and meditation, I was eager to create connections between these concepts and my artistic dance practice. Delving into improvisation and shortly thereafter being introduced to social therapeutics helped me develop a vision to do just that.

As a young dancer, I experienced improvisation as uncomfortable and purposeless, and I didn't care much for it. At that time, I was drawn by technique and prowess, pop music and set choreography. In college, my curiosity for improvisation steadily grew, but it wasn't until I was done with my bachelor's and attended an improvisation intensive with the dance collective Lower Left, that something shifted. I was able to see and experience for the first time the true vulnerability needed to improvise, the trust that needed to be built between partners and ensemble members, and the attention I needed to develop in order to build with others. I was able to feel the presence that I had experienced in meditation - the same awareness I had been fostering sitting on a cushion-now in motion.

Since then, I have delved into improvisation with different mentors and techniques and continue to discover more about myself, about relating and about collective creativity-how it develops and what it 
community work was highlighted when I was introduced to social therapeutics. I became aware of the importance of fostering listening, acting selflessly and building with others (abilities that are inherent in ensemble improvisation and that develop through this work) in multiple settings. When I started to bring this work into the community, I further appreciated the abilities that are present in my artistic practice. I was able to clearly see that these were not common skills and through listening to the experiences of participants unfamiliar with improvisation, my eyes were opened to the importance of incorporating these values into daily life.

\section{Act II: Coming Together}

It was in that first COLAB class that we met. We were both intrigued by this new activity of improvising across disciplines and were thirsty for more. We started collaborating as a duo through rigorous play, philosophizing and improvising together. In our first year, we got together two to three times a week for several hours at a time to develop a common language using music and dance. We shared common aspects of our individual disciplines that we were then able to adapt into our interdisciplinary work. Our starting point was recognizing the analogy between sound and movement, and silence and stillness, exploring the possible relationships between these while improvising. Does there need to be movement every time there is sound and vice versa? Is the quality of the sound matching the quality of the movement? Are they dissonant? When does that change and why? What is the relationship between the silence and stillness and the content of what it is being created? How does that space give the improvisation relevance? How does the attention to stillness/silence help us disrupt timing? How does that emerge interdisciplinarily—not only for Chris within the music and for Sandra Paola within the dance?

This interdisciplinary practice helped us reframe the way we each thought about improvisation. Our explorations caused me (Chris) to reevaluate what it meant to perform and be a performer. Focusing more on the activity of improvising collectively, rather than the content of my improvisation, opened up a space for me to be creative in a way that brought me back to my early, uninhibited experiences improvising at the piano in which I would lose myself in the moment. Because I, as a musician, was improvising with a dancer, I experienced more freedom in musical content (melody, harmony, rhythm), enabling me to focus more on relating with my fellow performers in other settings. Connecting with the ensemble became my primary concern, rather than "making the changes" (i.e. using the correct scale for a particular chord).

Sharing an interest in Buddhism and Eastern philosophies, we brought our individual meditative practices into our improvisation sessions. We started by simply sharing a meditation before improvising together and eventually began incorporating exercises that further explored the concepts of awareness and acceptance. Drawing from the Lower Left Collective's repertoire, "Inviting to be Seen," opened the door for us to exercise being fully present when witnessing our improvising partner, both in stillness/silence and in motion/sounding. In the exercise, after assigning roles of performer and witness, the performer stands in stillness with the intention of being open to being observed, and the witness traces with their eyes the performer's body with the intention of observing what is, and taking in as much detail without judgement. After switching roles, the same exercise is repeated with sound or motion. Not judging, of course, is incredibly difficult in our culture, which teaches us to label continuously. For us, the activity is about noticing those thoughts as they arise and observing them pass, maintaining our attention on what is experienced in the present moment-exactly the activity we do in our meditation practice. By design, "Inviting to be Seen" enables the performer to experience heightened vulnerability, while helping prepare the witness to radically accept what she/he is being offered without judging the content and/or quality.

Another activity, the "Orange Meditation," which was initially suggested by Jennifer Monson, became one of our most cherished practices. An everyday activity was transformed into an extraordinary experience just by giving it our undivided attention. The exercise consists of one person taking at least thirty minutes to peel and eat an orange while the second person witnesses in silence, both people being intentionally fully immersed in the experience. The time and attention required in the exercise enhances the texture, sounds, smell and taste of the orange to an almost unrecognizable point. Furthermore, both roles were important for us to question the aesthetic value of an improvisation and the definition of performance. The exercise of eating the orange had the same characteristics of an improvised performance: performing a task (or score), being aware of what was happening in the moment and 
responding to it, and being witnessed ... so, was it? This activity resonated with our use of mundane and pedestrian movement and non-traditional sound, which are important elements of our collaborative improvisation practice.

Beyond the opportunity that improvisation gave us to deepen our spiritual practice, these exercises helped us draw clearer connections on how developing awareness could be useful in everyday life. A clear example for me (Sandra Paola) was being able to leave an oppressive relationship. I spent hours both in meditation and improvising, focusing on listening to myself, my heartbeat, my breathing pattern, my thought processes, my emotionality-always giving in to what I heard/felt that I needed at each particular moment. It gave me the ability to be present in moments of conflict, to accept and build with what was happening without judging, and all the while remain equanimous. In this process, my life became the improvisation and in the practice, I was able to notice what I needed and wanted for my life.

As we developed our work, we came to realize that although we incorporated many elements of meditation in our improvisation practice, it was in improvised performance that we were able to practice focus, awareness, and acceptance in relationship to others. In contrast to meditation, collaborative improvisation is distinctly a social activity. We learn how to constructively respond to our surroundings, not just noticing them as we do in meditation. This includes other people, with their multitude of fluctuating experiences and responses, which necessitates a continuous reframing of our responses. Therefore, we concluded that because we are social beings, improvisation better enables us (people) to practice awareness (and other benefits of meditation) in our daily lives.

At the same time we were making these realizations, we encountered social therapeutics and were fascinated by the parallels between this methodology and our ensemble improvisation practice. We were particularly excited about its overt embracing of performance and improvisation as developmental tools for social change. There are four main tenets to the radical humanism of this methodology: (1) people are social beings-this alludes to our complexity and interconnectedness with others and the world, departing from the alienating view of the individual as a separate entity (2) people are performers - we are able to continuously create and recreate our lives; (3) people are improvisers-we all improvise to various degrees every day and are capable of being spontaneous and of dealing with the unexpected; and (4) people are revolutionaries - we are both determined by what is and what is becoming; therefore, we have the capacity to transform our current circumstances ("About Social Therapeutics"). By further contextualizing the transformative power of our improvisation work, social therapeutics fueled our desire to contribute to social change by using our experience as improvising performers.

We do most of our community work through ImprovISA, with the mission to empower communities through performance and improvisation. ImprovISA is based in El Paso, the western-most point of Texas, bordering Mexico to the south and New Mexico to the northwest about halfway between Houston, Texas and Los Angeles, California. This location, though geographically isolated, has created a strong binational cultural identity that encompasses the entire region. In addition to working with diverse populations in this city (which includes our students at the University of Texas at El Paso), we have the privilege to extend our reach across the United States border on a regular basis. We travel to neighbouring Ciudad Juarez across one of several bridges that go over the Rio Grande/Rio Bravo river, which, in this area, marks the physical border between the United States and Mexico. For improvising artists committed to community organizing and generating access to experimental performance, this location offers a unique environment for our artistic and community-based work.

ImprovISA runs ongoing programs, short-term projects, and one-time events that have grown out of our experiences interacting with and responding to the needs of communities on the border. Our work reflects our deep commitment to fostering human development through music and dance ensemble improvisation, and generating access to improvised and experimental performance. One of our programs, The Cattleya Project, aims to empower women and promote gender fluidity. This project grew out of Sandra Paola's interest in advocacy for women's rights and the heightened prevalence of violence towards women in Ciudad Juarez, including the high number of femicides perpetrated in the city in recent decades. This led us to begin a dialogue, and later collaboration, with Sin Violencia A.C.- - a shelter in Ciudad Juarez for women and their children escaping severe domestic violence- to give performance and improvisation-based workshops in order to create with them new ways of relating to their bodies, their children and other women in the shelter. Another ongoing program, knee-jerk, is a performance laboratory ensemble that grew out of our necessity to continue to develop and explore our methodology in practice. This El Paso-based ensemble brings together professional and amateur performers coming from dance, music, and theater backgrounds for regular rehearsals, workshops, and performances, allowing us to both develop our work in the studio and generate access to this aesthetic in different communities. This short video offers the reader an idea of how our work looks in practice: https://vimeo.com/174878550. 


\section{Act III: Collaborative Improvisation through the Lens of Social Therapeutics}

In our work, we focus on ensemble improvisation that is collaborative and interdisciplinary. In contrast to group work that results in individuals adding their own ideas together, when collaborating, we focus on the activity of co-creating something new. ${ }^{3}$ In our experience, interdisciplinary work pushes us to move beyond comfortable ways of relating to others, and, in doing so, ignites collective creativity. When blending movement and sound improvisation, we strive to be free from pre-determined roles traditionally ascribed to dance and music in the West-that is, the idea that "one [is] historically female, the other historically male; one of the body, the other of the mind; one seen, the other heard" (Goldman 56). One of the ways that we do this is by blurring the line between mover/sounder, fluidly moving between the two, and exploring ways in which we can see music and hear dance. For example, in knee-jerk, we continuously explore ways in which the movers/dancers make sound by moving, as well as vocalizing and ways that the sounders/musicians are recognized as visual components in the space and how they can move through it. Similarly, choreographer and improviser Martin Sonderkamp alludes to the benefits of role reversals when talking about his collaboration with bassist Christopher Williams: "[The reversals] subvert our cultivated ways of doing things and make us more alert. Instability sharpens our senses. The reversals also cause a certain sense of complicity, sometimes foolishness. Ultimately they open up a pathway for collaboration."

\section{"Building the Group"}

In collaborative improvisation practice, our interactions on stage are much more focused on active listening in order to create with what is palpable in the group. It is not a space for each of us as individuals to demonstrate what we can do; instead, it is an activity that requires us to constantly ask ourselves "how am I highlighting the group?" The motivation of every improvisation is to build the group, collectively creating something new with what emerges from that specific situation. Though this activity can be understood as good ensemble practice, it proves to be a challenge in other situations as it goes against all the years of competitive and individualistic training that our society promotes. When successful, it enables people to experience moments of deep connection with others and their surroundings, which in turn is what becomes deeply transformative for people's relationships in their daily lives. Using the social therapeutics language of 'building the group' to frame the understanding of this concept that we had experienced over and over again in improvisation helped us bridge our artistic practice and community organizing by giving us a common language to talk about both activities. It wasn't until we began introducing these concepts to others through workshops and presentations that we came fully to appreciate the value of improvisation as a vehicle for social change.

When we refer to "building the group," we are talking about the fundamental activity of social therapy. In addition to understanding people as social beings who create their lives together, social therapy highlights the dialectical relationship between the individual and the groups they inhabit (LaCerva and Helm 181). To illustrate better how this group unit works in social therapy, we look to Dr. Lois Holzman, the Director of the East Side Institute for Group and Short Term Psychotherapy, ${ }^{4}$ and the long-time collaborator of social therapy's creator, the late Dr. Fred Newman:

In most group therapies, the group serves as a context for the therapist to help individuals with their emotional problems. In social therapy, the group - not its individual members-is the therapeutic unit. Clients who come together to form a social therapy group are given a task-to create their group as an environment in which they can get help. This emphasis on group activity is a collective, practical challenge to the assumption that the way people get help therapeutically is to relate to themselves and be related to by others as individuals, complete with problems and with inner selves. It is in groups that a person's felt experience of being the center of the universe (that nothing else is going on in the world except how one is feeling) can be most effectively challenged. (Holzman 23)

When building the group, whether it is in an ensemble improvisation or in social therapy, the most important element is not the content. Rather, there is a powerful shift of focus to the human interaction (LaCerva and Helm 181). It is not what is being done by the individual performers that is significant, but how they relate to each other. What is being created is not as important as how it is being created. Drawn from improvisational theater, the concept of "yes and" is a great way to put this into practice. In her book improv wisdom, Patricia Ryan Madson suggests that "the spirit of improvising is embodied in the notion of "yes and" and explains how the "agreement begins the process" and "what comes next is to add something or develop the offer in a positive direction" (30). For example, if the offer is "it was great to see you at the show last night," a 'yes and' answer could be "yes, and it was great to see your kids again," rather than saying "what show?" With dance and music improvisation, the "yes and" process is much more abstract, leaving room for a wide variety of building responses and making it more difficult to describe. Nonetheless, a focus on this activity helps ensemble members move beyond merely reacting to stimuli, which would lead to a sort of "Mickey Mousing" effect where the sound and movement would mimic one another in a literal way, as in a Mickey Mouse cartoon. Instead, individual volitions come about in response to other ensemble members' actions, causing the 
spontaneous composition to evolve gradually over time or shift abruptly or a combination of the two. The effect of this plasticity causes the resulting performance to take many forms, reshaping itself organically. This can be seen in this video of an improvisation by Koan, a music and dance ensemble in which we both perform (https://vimeo.com/157746851). This improvisation begins with a punctuated rhythmic pattern from the piano (the offer), which is then built upon at 0:11 with a lyrical, arco bass melody (the first response). This bass entrance contextualizes the original offer by complementing and expanding the realm of the improvisation, instead of reacting by mimicking the rhythms of the piano. The piano and bass, combined, become an offer to which the dancer responds. Starting at 0:17, her slow movements contrast the forward momentum of the sound, again building upon the offer by adding something new. The addition of the saxophone at 0:27 increases the intensity of the sonic elements, further highlighting the contrast between the musicians and the dancer. The musicians remain in a motivic field, repeating and developing, which gradually pulls the dancer toward that intensity, both in movement quality (range of motion, increase in speed, contrasting dynamics, change in levels and tension in the body) and physical proximity to the musicians. The intensity level is brought down at 1:32 by the dancer's change in movement quality, leading to a pause. This invites a change in volume and intensity of the musicians at $1: 38$, starting with the saxophone and eventually leading to a bass solo. This sequence demonstrates the cyclical nature of overlapping "yes and" loops, which gives the group a life of its own: it becomes the improvising unit, just as it does in social therapy. Musician and educator Stephen Nachmanovich gives a similar description of an ensemble of musicians working as an "organism that has its own nature and its own way of being," which he terms "group personality" or "group brain" (94-5).

In the activity of improvising together, the ensemble creates and develops the form spontaneously and collectively: "the group that develops must continuously create and recreate the form of (group) life; that is, the group activities must include the continuous making of the group" (Newman and Holzman, Unscientific 165). The way in which an improvising ensemble creates itself and the form simultaneously can be seen as the practice of Newman/Holzman's tool-and-result methodology, which was derived from Russian psychologist Lev Vygotsky's radical break from the tool-for-result scientific paradigm of the early 1900s. The structure of the ensemble does not lead the improvisation, but instead the ensemble and the improvisation have a dialectical relationship that is "an activity (a 'search') that generates both tool and result at the same time and as continuous process" (Holzman 9).

\section{Perspectives on Listening: Connecting Meditation, Improvisation and Social Therapeutics}

The connection between meditation and improvisational practices has been widely experienced and well-documented (Nachmanovitch 19; Hay 64; Goldman 58; Zaporah 22; Oliveros xvii). As daily practitioners of Vipassana meditation, ${ }^{5}$ we take this practice to heart when we improvise. As improviser Kent De Spain mentions, "improvisation is a way of being present in the moment, and your awareness of yourself within that moment both challenges and refines your presence in each subsequent moment" (27). In our experience, the activity of building the group inspires each group member to strive toward letting go of their ego and acting selflessly, continuously bringing their attention back to the collective in the present moment. Building the group selflessly depends upon the awareness of ensemble members, and we find that further developing our awareness in our practice relies on enhancing our listening skills.

Pauline Oliveros has pointed to many connections between awareness and listening through her Deep Listening practice, which for her was about learning to expand perception (xxiii). This activity becomes a form of meditation, directing attention to the "interplay of sounds and silences" (Oliveros xxiv). Paying close attention to all perceivable sounds (not just "musical"), it is "intended to expand consciousness," simultaneously extending the listener's perception outward to the entire space/time continuum, as well as focusing on singular sounds (Oliveros xxiv). As movement-sound improvisers, listening deeply and actively goes beyond aural listening, and thus we bring kinesthetic listening practices into our work. We refer to kinesthetic listening as the ability to sense and listen to other bodies through spatial awareness and touch. We investigate and expand this ability mainly through Contact Improvisation, ${ }^{6}$ Ensemble Thinking, and Action Theater, where we play with giving and taking weight, counterbalance, movement structures, seeing/being seen, framing actions, among other things. ${ }^{7}$ For example, one of the first exercises we use to enhance an ensemble's spatial awareness is called "One Idea." Drawn from Ensemble Thinking, the objective of this exercise is "to have all the individuals agree on and exhibit one spatial idea ... [and] to create a shared notion of compositional form" (Martin 13). Dr. Nina Martin, the key developer of this form describes the exercise as follows:

One person runs somewhere in the room and takes a position in the space, lying, sitting, or standing. Everyone else runs in sequentially to build on that idea. Persons 1, 2 and 3 are very important because they establish the spatial concept, such as a diagonal line, circle, random spacing, or any other idea they come up with. Persons 4, 5, 6 and 7 have less choice, as the idea is already formed, and it is their job to recognize and support it. If everyone gets into a line facing forward and one person faces backwards, then the viewer's awareness is drawn to the variation and away from the "one idea" because the human eye is drawn to 
variation. The variation is not a problem, but does the dancer understand that he has introduced an idea (opposite facing) that is adding complexity? Often the choice to complicate a composition is made unconsciously. (13)

Our goal in using exercises like this is to help members assume "physical coordination and union with all things," which extends beyond the ensemble into the floor, the walls, audience members, and objects in the space (Smith 155). In other words, the body is "no different than the space it is moving in and the sound it is moving to," and when improvising with other people, bodies and sounds become extensions of each other (Zaporah 23).

When we view listening through the lens of social therapeutics, we focus on listening as a completing activity:

Listening is the quintessential completing activity; it's what the other person or people (whoever is not speaking) has to do, or give, for communication to take place. By "completing," l'm not talking about finishing, or putting closure on, what someone has said, but about continuing the activity-initiated by the speaker-of communicating. (Newman Let's 34)

For Newman, listening is the prerequisite for building the group_-it is the medium of building (qtd. in Holzman and Mendez 114). He goes further to describe a deeper level of communication: listening actively and attentively so that a person does more than "simply hear the words being said." Instead, they "actually experience the other person as a whole human being engaged in the effort to communicate_-to create-with language being the instrument" (Let's 34).

\section{Act IV: Performance, the Cornerstone of Social Therapeutics}

"For it is only in performing - a human skill which for most of us is left to atrophy after early childhood-that we can be who and what we are not." (Newman and Holzman, Unscientific 165)

No concept surfaces more in social therapeutics' literature than that of performance-our human capability to be other than who we are (Newman qtd. in Holzman and Mendez 83). Over the past twenty years, "social therapeutics has understood the core of its method to be performance and its core activity as that of bringing performance and play to daily life" (Friedman and Holzman 282). Performance is developmental because it "is the socialized activity of people self-consciously creating new roles out of what exists for a social performance" (Newman and Holzman, Vygotsky 83). We can all experience this to an extent when we dress up and change our behavior for a special event (e.g. an interview, a wedding, a ceremony)—we are giving a different performance of ourselves. One of the greatest teachings we have learned from social therapeutics is the fundamental fact that we are a performative species; we can use performance to free ourselves from socially prescribed roles, and, in this way, create new ways of being. From his experience as a theater director and playwright in the Castillo Theatre, ${ }^{8}$ Newman spoke about performance as a socially transformative activity:

We understand performance very broadly. From our point of view performance might have nothing to do with being on the stage. We think you can perform at home, at work, in any social setting . . With the proper kind of support, people discover that they can, that we can, do things through performance that we never thought we could do ... In a sense, we're trying to broaden each person's notion of "what you're allowed to do." We think that's a developmental experience (qtd. in Friedman, "Performance").

However, for most people, when they see artists singing, acting, or dancing, performance seems far removed from their reality, something that can only be done on stage by a professional. They fail to realize that we are all "biologically wired" to play and therefore perform (Bailey 138). Enabling people to reconnect with their innate ability to perform is our main goal when working with communities. This is paramount for society to develop, and, in order for social equity to be possible, we need to advocate in particular for the development of those who are in any way disenfranchised. Our social change movements having been overwhelmingly (mis)guided by ideology and led by confrontation, and have left our world deeply divided and stuck. Performance-the human capacity to perform-offers a way out, an opportunity to create new tools to address our social problems in new ways (Friedman, "Good-Bye" 126).

Our favorite way to introduce people to performance is through the "gibberish conversation," a game where they introduce themselves to one another. As we give instructions we mention different details they can include, such as their name, profession, where they were born, and their favorite food, to help them prepare a loose script. Right before we get ready to start the game, we introduce the final performance direction: they will do this in gibberish-in other words, a made-up language. This instruction is initially met by gasps, giggles and other signs of uncomfortableness, but upon reflecting after the activity is completed, participants see that a small change in how 
they are introducing themselves has a great impact on the quality of their interaction. It is therefore, by using play that participants experience the developmental aspect of performance-being who they are (because we never cease to be that) and who they are not (because most people don't introduce themselves in gibberish on a regular basis) — without knowing or naming it as a performance.

\section{Learning, Play and Development}

In social therapeutics, the idea of being who you are and who you are not illustrates the connection between performance and development - it is through performance that we develop. Development, as it is thought of in social therapeutics, is "a dialectical, continuously emerging socio-cultural-historical collective activity"; it is what we create through our capacity to perform (López Ramírez 30). This idea stems from Vygotsky's zone of proximal development (ZPD), a concept he developed through his investigations on the relationship between learning and development. Departing from other opinions of his time, Vygotsky pointed to the fact that the difference between what a child accomplishes by her/himself and what she/he can accomplish in collaboration with an adult or older peer is limited and varies from child to child. This showed "Vygotsky's concern with the character of the relationship between 'matured' and 'maturing' processes," emphasizing that what a child is currently in the process of learning (not just what she/he has already learned) is part of her/his level of development (Newman and Holzman, Vygotsky 45). Perhaps a simpler definition of ZPD is given by Newman and Holzman: "we think a ZPD is simply a form of life in which people collectively and relationally create developmental learning which goes beyond what any individual in the group would learn on her or his 'own'” (Unscientific 164). Furthering his theory of development, Vygotsky makes a distinction between the ZPDs created in everyday non-play situations and the ZPDs created in play:

play creates a zone of proximal development of the child. In play a child always behaves beyond his average age, above his daily behavior; in play it is as though he were a head taller than himself ... Though the play-development relationship can be compared to the instruction-development relationship, play provides a much wider background for changes in needs and consciousness. Action in the imaginative sphere, in an imaginary situation, the creation of voluntary intentions, and the formation of real-life plans and volitional motives - all appear in play and make it the highest level of pre-school development. The child moves forward essentially through play activity that determines the child's development. (qtd. in Newman and Holzman, Vygotsky 81)

Newman and Holzman furthered Vygotsky's theory by recognizing that development is something that happens throughout our lifespan and does not stop after childhood. They refer to Vygotsky's concept of being "a head taller" as performance "in the theatrical sense of the word" (Holzman 18). It is a space that allows for "improvisational developmental learning" where "the process of learning and the product of learning are created together" (Newman and Holzman, End 126; Holzman 18). Therefore, the reason performance is of utmost importance for our development as human beings is that it brings back the developmental learning that we find in childhood play beyond our childhood years. Sally Bailey speaks directly to this from her experience as a theater artist and drama therapist: "I have found that dramatic play is essential for people of all ages . . . it provides a safe haven in which players can rehearse skills and behaviors that transfer directly to performance on all of life's stages" (137). Newman reminds us that the stage, too, was something we (as the human species) created and that "we have the capacity to create environments in which we can perform. We can create stages anywhere" (qtd. in Holzman and Mendez 83). Therefore, people need to keep creating 'stages' throughout their lives in order to keep developing; the stage is our adult playground.

Creating these 'stages' in our work takes many forms that depend on the population with whom we are working. For example, one of the members of The Cattleya Project was able to build with a request from the women in the shelter to teach a Zumba class, transforming it into a performative developmental experience. After teaching them a few steps, she divided the women into small groups and gave them about ten minutes to create their own Zumba routine. When they were done, she had each group stage an improvised "Zumba instructor performance," where they had to embody the confident, upbeat, energetic, excited and coordinated qualities of an instructor while they led the rest of the group through the steps they had put together. As she demonstrated how a Zumba instructor may act, the women were invited to perform 'a head taller' and pretend they had the confidence, coordination, and energy to teach a Zumba class. There was a profound change in the posture, energy, and mood of the women when they followed the performance direction to pretend to be someone else. We all have that outstanding creative capacity, and "creative inspiration is not the property only of certain special people like professional artists" (Nachmanovitch 183). Therefore, with ImprovISA, we purposely search to perform and workshop with a wide variety of people: at-risk youth, women escaping severe domestic violence, community organizers, non-profit organizations, homeless youth, students, and academics. Furthermore, bringing artists together with these groups when creating performances allows for an environment that is enriched with diverse backgrounds, experiences, ages, and abilities; this environment boosts 
everyone's learning potential, given that, as Newman suggests, "we learn collectively and by our interrelationships with people at different learning levels" (qtd. in Holzman and Mendez 69).

\section{Act V: Everyday Life as a Collaborative Improvisation}

The practice of collaborative improvisation, because of how relationships between performers continuously evolve, creates a play space that can be viewed as a microcosm for other groups and society as a whole. As Newman has stated, "from the point of view of development ... we are better off relating to life as continuous happenings, continuous emerging processes, complex social activity, rather than as things happening to things" (qtd. in Holzman and Mendez 58). When teaching or facilitating a workshop, our aim is to enable participants to experience creating collectively and to facilitate dialogues and reflections so that they can see the applicability of these activities in the rest of their lives. Similar to how improvisation challenges established aesthetic frameworks in both music and dance, the capacity to improvise and perform in everyday life challenges the ways we are used to relating to each other. As Nachmanovich states, "collective free improvisation in the performing arts, music, dance, and theater invites us into whole new kinds of human relationships" (98-9). This is why, similar to social therapy, we view the experience of collective improvised performance to be a radical way of creating new ways of relating to others and the environment. We work with participants to find new ways of being and responding, first in performance practice and then in everyday life. For example, we use an Action Theater exercise to highlight the innumerable ways to respond to a particular action in an improvisation. We pair participants up and assign them the roles of 'doer' and 'responder.' The 'doer' performs an action and the 'responder,' using movement and/or sound, responds. This is repeated with the 'doer' performing the same action eight, ten, twenty times and the 'responder' having to come up with eight, ten, twenty different responses. While exploring the many ways to respond, it is important to bring participants' attention back to building with their partner by focusing on responding in a "yes, and" way to the given offer. After everyone gets a turn in both roles, we proceed to have a discussion where we invite participants to reflect on instances in their lives where it would be beneficial to have multiple ways to respond. A situation we have discussed with our all-women workshops is how to respond to unwanted comments about their appearance. The question becomes, how can you relate to this interaction as an improvisation and treat the comment as an offer with which to build? For example, if a man approaches a woman and asks, "Why don't you wear dresses more often?" Rather than shutting down the offer, a building response could be "You're right, next time, let's both wear dresses!" One more thing that is important in this instance, is that building responses always need to be honest, rather than sarcastic. Therefore, if your partner accepts your offer of wearing a dress, you should be willing to accept his offer.

For us, bringing improvisation into our everyday lives also means incorporating it in the way we design and implement ImprovISA's workshops, presentations, and programs. In our experience, this is necessary in order for community building to be possible as it enables us to adapt, listen, and craft together with everyone present. We put this into practice soon after beginning our first binational program in collaboration with Ccompaz. During the first few months of this program, we learned that we couldn't focus on interdisciplinary improvisation (which was our original plan) with music students who could not yet play basic melodies, chords, and rhythms - an important outcome set by the Ccompaz administration. This led us to focus solely on developing their music abilities for the remainder of that semester and eventually, to develop a separate dance program while redesigning their music curriculum to incorporate more improvisation. We also use improvisation in the building of knee-jerk, recognizing that the creation of the ensemble happens continuously within and outside the performance, having a significant impact on the group and individual members. This became evident when we first started the ensemble, as one of our members was a single mother without the means to find childcare for her three-year-old daughter. After several weeks of her daughter testing boundaries while other ensemble members tried to ignore the disturbance, we decided to open up a conversation about how we could build with the situation instead of treating it as a problem. How could the child become a creative source and opportunity? How could we all share the responsibility of attending to the child so that the mother would be able to participate equally in rehearsals? The result was to involve the child in some of our activities, leading many ensemble members to use her innate creativity as inspiration, and all of us collectively taking care of the child's needs and safety. Bringing awareness to how the child affected the ensemble brought us closer together through a shared responsibility of building the group and organizing our rehearsals.

\section{Coda}

Building our work is a collaborative improvisational performance-we are in constant flux, continuously emerging and becoming. In the words of Newman and Holzman, we are a development community, one that is "continuously, endlessly, making itself" (Unscientific 159). While developing our artistic and community work, we continue to draw inspiration from collaborative improvisation, social therapeutics' radical humanism, and the connections between the two. Collaborative improvisation, as we understand it, necessitates the building of the group and recognizing that people are social beings, two fundamental aspects of social therapeutics. This focus, while interacting with others in and outside improvised performance, enhances listening and expands our awareness to other people and our 
surroundings. Social therapeutics also helps us contextualize performance as a developmental tool; it is through performance that people are able to be who they are and who they are not, continuously recreating their lives. Furthermore, through improvisation we can offer communities a way to create new realities, to develop new ways of relating, and continuously to be who they are becoming by enhancing their capacity to be spontaneous and deal with the unexpected. This offers a revolutionary alternative to the perpetuation of societally prescribed roles that often involve cycles of violence, neglect, and despair, and thus allows us to transform our current circumstances.

We can no longer remain ignorant of the ethical implications of improvisation on social justice, and for us, part of this responsibility includes opening access to this work. Working with the communities on the border has made us recognize and question why the people we know who practice this performance form overwhelmingly come from white, progressive, middle-class backgrounds, given the fact that improvisation has been historically prevalent in African and indigenous music and dance. It is because of this recognition that we are pushing for wider access to experimental improvisation in this area. Two organizations that have influenced ImprovISA's development-the International Institute for Critical Studies in Improvisation and the All Stars Project-have openly recognized the impact of improvisation on social change and have been doing work like this throughout Canada, the United States, and beyond, as have many others.

Building on their offers, ImprovISA's practice marries the artistic and the developmental through the constant dialogue between social therapeutics and the experimental aesthetics of improvised performance. We expand people's aesthetic frameworks and encourage them to see themselves as creators by breaking the belief that they need to look, move, act or sound a particular way to be able to create and perform. Our rigorous focus on explorative process and collaborative improvised performance allows trained and untrained artists alike to find new ways of relating to art and see its relationship to development. It is through this practice that we invite others to improvise new realities and create change in themselves and the world.

\section{Notes}

1 This methodology was developed in the mid-seventies by a group of social workers, artists, therapists, and community organizers in New York City, and it is now being practiced in different iterations and contexts around the world.

${ }^{2}$ ImprovISA is building on the work we did with in2improv; you can visit www.in2improv.org to learn about our previous work. For more information on ImprovISA, visit www.improvisationandsocialaction.org.

${ }^{3}$ For more information on the difference between additive group work and collaborative work, see Sawyer (2007).

4 The East Side Institute for Group and Short Term Psychotherapy is the main hub and training center for social therapy (as a therapeutic practice) and social therapeutics (as a methodology). For more information, go to www.eastsideinstitute.org.

${ }^{5}$ Vipassana meditation, also known as insightful meditation, is a Buddhist meditation technique mostly practiced in the Theravada tradition. For further reading, see Sayadaw (2010).

${ }^{6}$ For more information on Contact Improvisation, see Novack (1990).

${ }^{7}$ A further description of Ensemble Thinking can be found at www.ensemblethinking.com and Martin (2007). More information on Action Theater can be found at www.actiontheater.com.

${ }^{8}$ The Castillo Theatre is a community-based theater that is born out of the same developmental movement of social therapeutics. For more information, go to: www.castillo.org and www.allstars.org.

\section{Works Cited}

Bailey, Sally. "Play as a Staging Ground for Performance and Life." Play and Performance: Play and Culture Studies, Vol. 11, edited by Carrie Lobman and Barbara E. O'Neill, UP America, 2011, pp. 137-54. 
De Spain, Kent. "The Cutting Edge of Awareness: Reports from the Inside of Improvisation." Taken by Surprise: A Dance Improvisation Reader, edited by Ann Cooper Albright and David Gere, Wesleyan UP, 2003, pp. 2738.

Friedman, Dan. "Good-Bye Ideology. Hello Performance." Topoi: An International Review in Philosophy vol. 30, no. 2, 2011, pp. 125-35.

---. "Performance as Revolutionary Activity: Liminality and Social Change." Body, Space \& Technology Journal vol. 1, no.1, 2000, www.bstjournal.com/vol0101/index.html. Accessed 17 Dec. 2017.

Friedman, Dan and Lois Holzman. "Performing the World: Performance Turn in Social Activism." Performance Studies in Motion: International Perspectives and Practices in the Twenty-First Century, edited by Atay Citron, Sharon Aronson-Lehavi, and David Zerbib, Bloomsbury, 2014, ch. 20. Kindle File.

Goldman, Danielle. I Want to Be Ready: Improvised Dance as a Practice of Freedom. U Michigan P, 2010.

Hay, Deborah. my body, the buddhist. Wesleyan UP, 2000.

Holzman, Lois. Vygotsky at Work and Play. Routledge, 2009.

Holzman, Lois and Rafael Mendez, eds. Psychological Investigations: A Clinician's Guide to Social Therapy. BrunnerRoutledge, 2003.

LaCerva, Christine and Chris Helm. "Social Therapy with Children with Special Needs and Their Families." Play and Performance: Play and Culture Studies, edited by Carrie Lobman and Barbara E. O'Neill, vol. 11, UP of America, 2011, pp. 180-97.

López Ramírez, Sandra Paola. "Ensemble Improvisation as a Developmental Tool." Moving On, vol. 11, nos. 3-4, 2014, pp. 28-31.

Madson, Patricia Ryan. improv wisdom: Don't Prepare, Just Show Up. Bell Tower, 2005.

Martin, Nina. "Ensemble Thinking: Compositional Strategies for Group Improvisation." Contact Quarterly, vol. 32, no. 2, 2007, pp. 10-15.

Nachmanovitch, Stephen. Free Play: Improvisation in Life and Art. Penguin Putnam, 1990.

Newman, Fred. Let's Develop: A Guide to Continuous Personal Growth. All Stars Project, 2010.

Newman, Fred and Lois Holzman. Lev Vygotsky: Revolutionary Scientist. Psychology P, 2014.

---. The End of Knowing: A New Developmental Way of Learning. Routledge, 1997.

---. Unscientific Psychology: A Cultural-Performatory Approach to Understanding Human Life. iUniverse, 2006.

Novack, Cynthia. Sharing the Dance: Contact Improvisation and American Culture. U Wisconsin P, 1990.

Oliveros, Pauline. Deep Listening: A Composer's Sound Practice. iUniverse, 2005.

Sawyer, Keith. Group Genius: The Creative Power of Collaboration. Basic Books, 2007.

Sayadaw, Chanmyay Ven. Talks on Meditation given in the Blue Mountains. Blue Mountains Insight Meditation Centre, 2010.

Smith, Nancy Stark. "A Subjective History of Contact Improvisation: Notes from the Editor of Contact Quarterly, 19721997." Taken by Surprise: A Dance Improvisation Reader, edited by Ann Cooper Albright and David Gere. Wesleyan UP, 2003, pp. 153-73. 
Williams, Christopher and Martin Sonderkamp. "Where You End and I Begin: Cognition and Continuity in Experimental Improvised Music and Dance." Critical Studies in Improvisation, vol. 8, no. 2, 2012, www.criticalimprov.com/article/view/2144. Accessed 17 Dec. 2017.

Zaporah, Ruth. "Dance: A Body with a Mind of Its Own." Taken by Surprise: A Dance Improvisation Reader, edited by Ann Cooper Albright and David Gere, Wesleyan UP, 2003, pp. 21-4. 